Research Paper:

\title{
Evaluation of Prognostic Factor and Pain Intensity Following Thoracolumbar Disc Surgeries
}

\author{
Ali Meshkini ${ }^{1}$ (D) , Firoz Salehpour ${ }^{1}$ (D) Farhad Mirzaei ${ }^{1}$, Saeid Rouhi ${ }^{1}$, Mandana Ghafouri Rahimabadi ${ }^{2}$, Seyed Ahmad Naseri Alavi ${ }^{*}$ (CD)
}

1. MD., Department of Neurosurgery, Faculty of Medicine, Tabriz University of Medical Sciences, Tabriz, Iran

2. School of Medicine, Tehran Medical Branch, Islamic Azad University, Tehran, Iran

$\begin{aligned} & \text { Use vour devict to san } \\ & \text { and read the article online }\end{aligned}$
Cittation Meshkini A, Salehpour F, Mirzaei F, Rouhi S, Ghafouri Rahimabadi M, Naseri Alavi SA. Evaluation of Prognostic Factor
and Pain Intensity Following Thoracolumbar Disc Surgeries. Iran J Neurosurg. 2018; 4(3):139-148. http://dx.doi.org/10.32598/
irjns.4.3.139

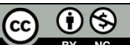

Article info:

Received: 25 December 2017

Accepted: 09 April 2018

Available Online: 01 July 2018

Keywords:

Pain, Disc surgery, Visual Analog Scale, Prognosis

\section{ABSTRACT}

Background and Aim: Thoracolumbar Junction (TL) is a transitional zone between the rigid thoracic spine and the flexible lumbar spine. Diagnosis and treatment of herniated discs pertaining to this spinal region is difficult owing to its unique anatomy has challenges to spine and orthopedic surgeons and neurosurgeon. There is not definite approach to TL surgeries, with different controversies surrounding each. Therefore, the outcomes and complications associated with different approaches were examined in this study.

Methods and Materials/Patients: After being approved by ethics committee of Tabriz university of medical sciences Forty-nine patients undergoing TL disc surgeries during 2012-2016 were studied in three Iranian hospitals, i.e. Imam Reza and Shohada hospitals in Tabriz, and Azarbaijan Hospital in Urmia. Patients were examined in terms of gender, age, BMI, surgical level, number of surgical surfaces, lower extremity pain, back pain, paresthesia of limbs and perineum, weakness of lower limbs, sphincter disorder, surgery types including laminectomy+festectomy+discectomy with or without Fusion and with or without Fixation.

Results: From Forty-nine patients, 22 patients were male and 27 were female. The mean age was $46.75 \pm 6.83$. Nine patients had severe lower extremity pain (VAS $\geq 6$ ) and 40 patients had mild pain (VAS<6), which corresponded to postoperative limb pain: 24 patients reported severe pain and 25 mild pain, which lasted over 6 months. There was no significant relationship between fusion and lower extremity pain.

Conclusion: Based on the results of this study, which examined the factors affecting prognosis and severity of pain after thoracolumbar discs, spinal cord manipulation should be avoided due to limited circulation of the spinal area.

\section{* Corresponding Author:}

Seyed Ahmad Naseri Alavi, MD.

Address: Department of Neurosurgery, Faculty of Medicine, Tabriz University of Medical Sciences, Tabriz, Iran

Tel: +98 (911) 6137353

E-mail: dr.arsalan2010@gmail.com 


\section{Highlights}

- Due to limited circulation of the spinal area spinal cord manipulation is one of the most important factors affectng prognosis and severity of pain after thoracolumbar discslt is better to use posterior approach and fixation with a pedicure screw in patents with hard discs, to discharge the disc and austerites, to reduce the severity of back pain.

- There were no signifcant correlatons between the variables, i.e. back pain and the three surgical methods based on lower extremity pain and surgical technique, dissectors and lower limb pain, dissectors and back pain, fusion and fxaton with back pain, fusion and fxaton with lower limb pain.

- Patients with a history of myelopathy should be more cautious than normal patients due to the higher rates of complications.

\section{Plain Language Summary}

Thoracolumbar Junction (TL) is a transitional zone in the spine area and vulnerable to trauma. However, TL is less susceptible to disk degeneration in comparison with the lower part of the spine. There are different approaches for TL operations with different outcomes. This study evaluated these approaches in 49 patients undergoing TL disk surgeries in three hospitals in Iran during 2012-2016. The patients were examined in terms of their gender, age, BMI, surgical level, number of surgical surfaces, lower extremity pain, back pain, paresthesia of limbs and perineum, weakness of lower limbs, sphincter disorder, and surgery types, including laminectomy plus festectomy plus diskectomy with or without fusion and with or without fixation. Based on the results of this study, in thoracolumbar disk operations, spinal cord manipulation should be avoided due to limited circulation in the spinal area.

\section{Introduction}

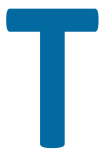

horacolumbar Junction (TL) is a transitional zone between the rigid thoracic spine and the flexible lumbar spine. It is the region where changes in the cauda equine occur. Moreover, it is vulnerable to trauma, and is less susceptible to disc degeneration in comparison with distal lumbar spine [1]. The prevalence of TL is about $1 \%$ and is common in adults, only $0.25-5 \%$ become symptomatic [2].

Various signs and symptoms related to thoracolumbar discs have been described. Owing to the prevalence of accidentally-detected discs in this area and its asymptomatic nature, the association of symptoms to a herniated disc is occasionally difficult due to the anatomical complexity of the cord and the presence of cauda equine and abundant neural roots [1].

Frequent traumas in adults are attributed to exercise, accident and the vulnerability of this region, which can lead to disc degeneration. However, $22-50 \%$ of symptomatic patients have a history of trauma [3]. Middleton and Teacher were the first to perform a thoracic disc herniation surgery in 1911 [4].
Herniation of thoracic discs was first explained by Key in 1938 who argued that the diagnosis and treatment of the discs of this region were difficult [5]. The treatment of this region, specifically the cause of calcified discs' exclusive anatomy, is a subject of discussion among spine surgeons. A lack of adequate method of dealing with this problem is also feel in orthopedic and neurosurgeries reference books [6]. There are no general criteria for choosing the best surgical method for thoracolumbar disc herniation [7] In different studies, various levels have been defined for this area from T10/T11 to L2/L3. However, thoracolumbar disc herniation in generally refer to T10/T11, T11/T12, T12/L1, and L1/L2 [1, 6].

Several surgical procedures and approaches are used to treat thoracolumbar discs. Anterolateral, lateral and posterolateral approaches have been initially performed on one side, providing no obvious view of the compressed lesions and dura mater of the other side. They have been reported to cause insufficient decompression and unintentional spine surgery [7]. However, many of such methods are technique-related and require entry into the chest. Surgeons have become increasingly proficient about these methods and the potential problems of each [8]. 
The symptoms of the patient include low back pain and lower extremity pain, radicular pain, limb paresthesia and perineum, lower limb weakness, urinary and fecal incontinence, urinary retention [1, 2, 5, 8-10]. In a study by Mulier \& Debois in 1997, the results of transthoracic, lateral, and posterolateral surgery were investigated in seven patients undergoing surgery between 1986 and 1993. They recommended a transthoracic method for the treatment of thoracic discs under T4, except for patients with a serious risk of pulmonary disease [8]. Therefore, the present study was designed to evaluate a comparison between the prognoses of different methods, identify the effective factors in the disc surgery of this area, and present a surgical procedure with the most favorable outcome and the least complications so as to allow a consensus to be reached for the treatment of discs in this area.

\section{Methods \& Materials/ Patients}

This study was carried out in two teaching hospitals of Imam Reza and Shohada in Tabriz as well as Azarbaijan Hospital in Urmia after being approved by ethics committee of Tabriz University of medical sciences, Iran. Patients visiting these centers underwent thoracolumbar disc surgeries during 2012-2017 based on inclusion and exclusion criteria.

They were provided with a checklist. Reviews of patient records, biographies, descriptions of surgery, follow-up phone calls and, if necessary, follow-up visits were included in this study. To avoid any kind of misconception, a unique code was assigned to each patient to prevent any medical error. Given the rareness of thoracolumbar discs and low sample size, all patients admitted to Imam Reza, Shohada, and Azerbaijan hospitals during sampling completed the checklist and were entered into the study. Patient information including age, gender, history of pain and preoperative organ weaknesses, preoperative back pain, preoperative sphincter disorder, the location of discs, disc types, the number of involved surfaces, surgery methods, and fusion or lack thereof were obtained from the analysis of the archives of the documents and the completed checklists.

Information about back pain, immediate postoperative limb pain, paresthesia and immediate postoperative limb weakness, post-operative sphincter disorder, and progress notes was filled in the questionnaire. The remaining items were completed through follow-up phone calls and, if necessary, follow-up visits to investigate potential fusions through a control graph.
Patients were examined in terms of gender, age, BMI, surgical level, number of surgical surfaces, lower extremity pain, back pain, paresthesia of limbs and perineum, weakness of lower limbs, sphincter disorder, surgery types including laminectomy+festectomy+discectomy with or without Fusion and with or without Fixation.

Using Visual Analog Scale (VAS), the severity of back pain and radicular pain was measured immediately after surgery and one month and six months following the operation. Organ weaknesses were measured through grading $(0,1.5,2.5,3.5,4.5,5.5)$. Finally, patients were scored from 1 to 10 depending on their postoperative quality of life. The inclusion criteria consisted of being over 18 years of age, expressing informed written consents, positive findings in Magnetic Resonance Imaging (MRI), and having undergone thoracolumbar disc surgery during 2012-2017.

The exclusion criteria consisted of having a history of disc surgery or fracture of the vertebrae irrespective of the spinal region, the presence of a disc in another spinal region, illiteracy or aphasia that prevents communication and obtaining of informed written consents, suffering from spinal stenosis in the thoracolumbar region without clear discopathy.

Data are given as Mean \pm SD. Kruskal Wallis H , Mann Whiteny $U$ and Chi-square test (categorical data) and Independent student t-test (numerical data) were used for comparisons. The effective factors in the groups were analyzed using regression analysis by adjusting the confounding variables to the OR report with a $95 \%$ confidence interval in SPSS V. 16 . In this study, $\mathrm{P}<0.05$ were considered statistically significant.

\section{Results}

Forty-nine patients were finally enrolled in this study, $22(44.9 \%)$ of whom were male and 27 (55.1\%) were female. The mean age was $46.75 \pm 6.83$ (Table 1 ). Table 2 shows disc level distribution in patients who have underwent surgery. From 49 patients, 9 (18.4\%) had severe lower extremity pain (VAS $\geq 6$ ) and 40 (81.6\%) had mild pain (VAS<6), which corresponded to postoperative limb pain: 24 (49\%) patients reported severe pain and 25 (51\%) mild pain, which lasted over 6 months.

More patients had visited by a physician for more than one year from the onset of symptoms (Table 3 ). Regarding to the severity of back pain, $3(6 / 1 \%)$ patients had severe pain (VAS $\geq 6$ ) and $46(93.9 \%)$ had mild pain $(\mathrm{VAS}<6)$ before surgery in comparison with those re- 
Table 1. Age distribution in patients enrolled to the study

\begin{tabular}{ll}
\hline Age & No. (\%) \\
\hline $20-30$ & $5(10.2)$ \\
\hline $30-40$ & $10(20.4)$ \\
$40-50$ & $14(28.6)$ \\
$50-60$ & $13(26.5)$ \\
$60-70$ & $5(10.2)$ \\
\hline
\end{tabular}

Table 2. Disk level distribution in patients enrolled to the study

\begin{tabular}{cc}
\hline Disk Level & No. (\%) \\
\hline T10/T11 & $5(10.2)$ \\
\hline T11/T12 & $7(14.3)$ \\
T12/L1 & $13(26.5)$ \\
L1/L2 & $14(28.6)$ \\
Two level & $10(20.4)$ \\
\hline & \\
\hline
\end{tabular}

porting postoperative back pain: 25 (51\%) experienced severe pain and 24 (49\%) mild pain (VAS<6), lasting for over 6 months.

From 49 patients, 48 (98\%) had paresthesia in the limbs and perineal area and $1(2 \%)$ had no paresthesia. Post-operative paresthesia was observed as follows: $37(75.5 \%)$ patients experienced paresthesia for over 6 months, 6 patients (12.2\%) had no paresthesia, 5 (10.2\%) had immediate postoperative paresthesia, and $1(2 \%)$ patient suffered from postoperative paresthesia until two weeks after the surgery, which was finally re-

Table 3. Numbers show times in a month patients visited by a doctor solved. Out of 49 patients, 23 (46.9\%) had soft discs and $26(53.1 \%)$ had hard discs and osteophytes.

Muscles force decreased for over 6 months (Table 4). According to Table 4, surgery did not have a significant effect on post-operative recovery of limbs power. Physician visits and surgical procedures before the reduction of limb power would lead to more favorable outcomes. From 49 patients who underwent operation, 11 (22.4\%) patients had a preoperative history of myelopathy, which caused the reduction of the power of lower extremities to less than 3.5, dependency on wheelchair, walking stick, or surrounding people in 10 (90.9\%) pa-

\begin{tabular}{cc}
\hline Time (Month) & No. (\%) \\
\hline$<1$ & $1(2)$ \\
$1-6$ & $12(24.5)$ \\
\hline 12 & $10(20.4)$ \\
\hline
\end{tabular}


Table 4. Lower limbs muscle force comparison before and after the surgery

\begin{tabular}{ccc}
\hline \multirow{2}{*}{ Muscle Force } & \multicolumn{3}{c}{ Number of Patients (\%) } \\
\cline { 2 - 3 } & Before Operation & After Operation \\
\hline 0.5 & $2(4.1)$ & $3(6.1)$ \\
\hline 1.5 & $2(4.1)$ & $2(4.1)$ \\
\hline 2.5 & $3(6.1)$ & $2(4.1)$ \\
\hline 3.5 & $9(18.4)$ & $6(12.1)$ \\
\hline 4.5 & $30(61.2)$ & $8(16.3)$ \\
\hline 5.5 & $3(6.1)$ & $28(57.1)$ \\
\hline
\end{tabular}

tients. Eight (72.7\%) patients had post-operative sphincter disorder. Patients were asked to give a score of 0 to 10 for the limitations they experienced in their overall lifestyle as a result of surgery. A score of 10 represented a great impact and a poor condition of life. Patient scores are showed in Table 5.

From 49 patients, 12 (24.5\%) patients underwent laminectomy, facetectomy, and discectomy (the first method), and 18 patients (36.7\%) underwent laminectomy, facetectomy, fusion, and pedicular screw fixation insertion without dissectors (the second method). Nineteen (19) patients underwent laminectomy, facetectomy, dissectors, fusion, and pedicular screw fixation (the third method).

From 12 patients who received surgical treatment with the first method, 8 (66.7\%) patients had mild backache $(\mathrm{VAS}<6)$ and $4(33.3 \%)$ had severe low back pain (VAS $\geq 6$ ) following surgery. From 18 patients who were surgically treated with the second method, 16 (88.9\%) had mild pain and 2 (11.1\%) had severe pain. From 19 patients who underwent surgical procedure with the third method, 17 (89.5\%) had mild pain and 2 (10.5\%) had severe pain. Those who had underwent dissectors reported a greater "mild back pain" that was not statistically significant $(P=0.18)$.

Mild back pain $(\mathrm{VAS}<6)$ was higher in those who underwent fusion, albeit not statistically significant $(P=0.66)$. Comparison of the level of back pain in terms of the applied surgical methods revealed no significant difference $(P>0.05)$ (Figure 1$)$. Regarding to postoperative lower limb pain using the first surgical method, 10 $(88.3 \%)$ patients had a mild pain (VAS<6) and $2(16.7 \%)$ had a severe pain (VAS $\geq 6$ ). From 18 patients who were surgically treated through the second method, 17 (94.4\%) had a mild pain and 1 (5.6\%) had a severe pain. From 19 patients who were surgically treated through the third method, 14 (73\%) had a mild limb pain and $5(26.3 \%)$ had a severe pain in the extremities. From a statistical analysis, the higher prevalence of pain in the second group was not significant $(P=0.23)$ (Figure 2).

Regarding the relation between dissectors and postoperative lower extremity pain, out of 18 patients with non-discectomy, $17(94.4 \%)$ had a mild pain and $1(5.6 \%)$ had a severe limb pain. From 31 patients with

Table 5. Distribution of life limitations after surgery ( 0 means good and 10 is more limitations)

\begin{tabular}{|c|c|}
\hline Grading (0-10) & No. \\
\hline $0-2$ & 8 \\
\hline $2-5$ & 11 \\
\hline $5-8$ & 14 \\
\hline 8-10 & 16 \\
\hline
\end{tabular}




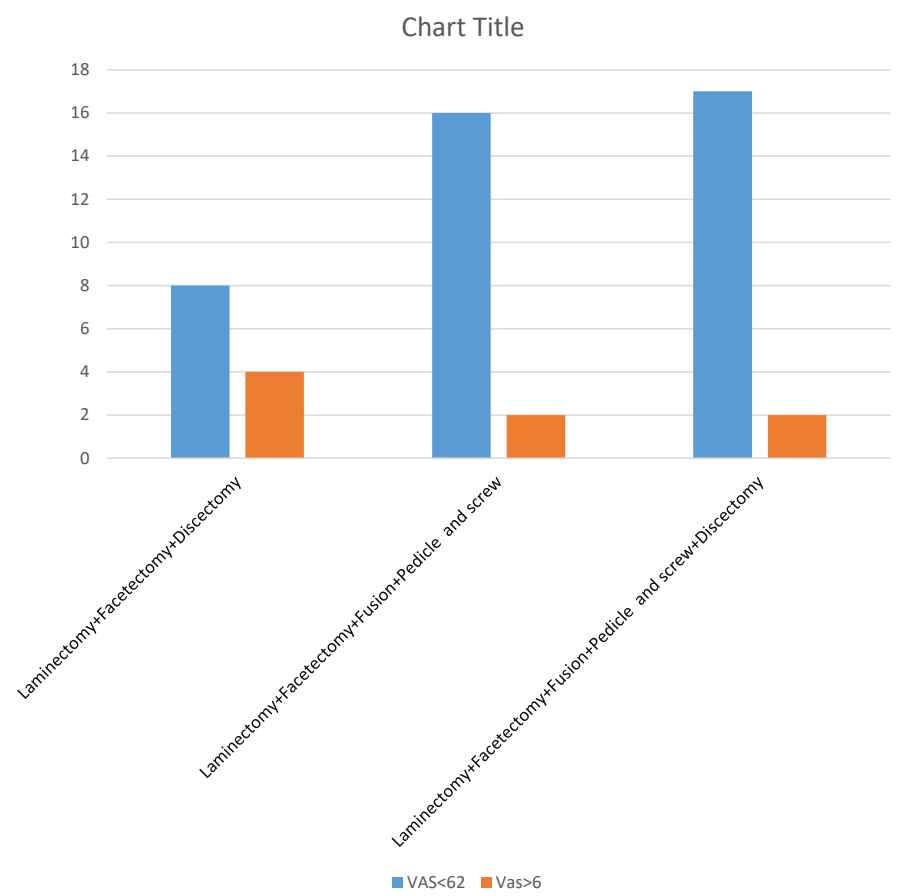

INS

Figure 1. Comparison of frequency of low back pain in three different method of surgery based on VAS score

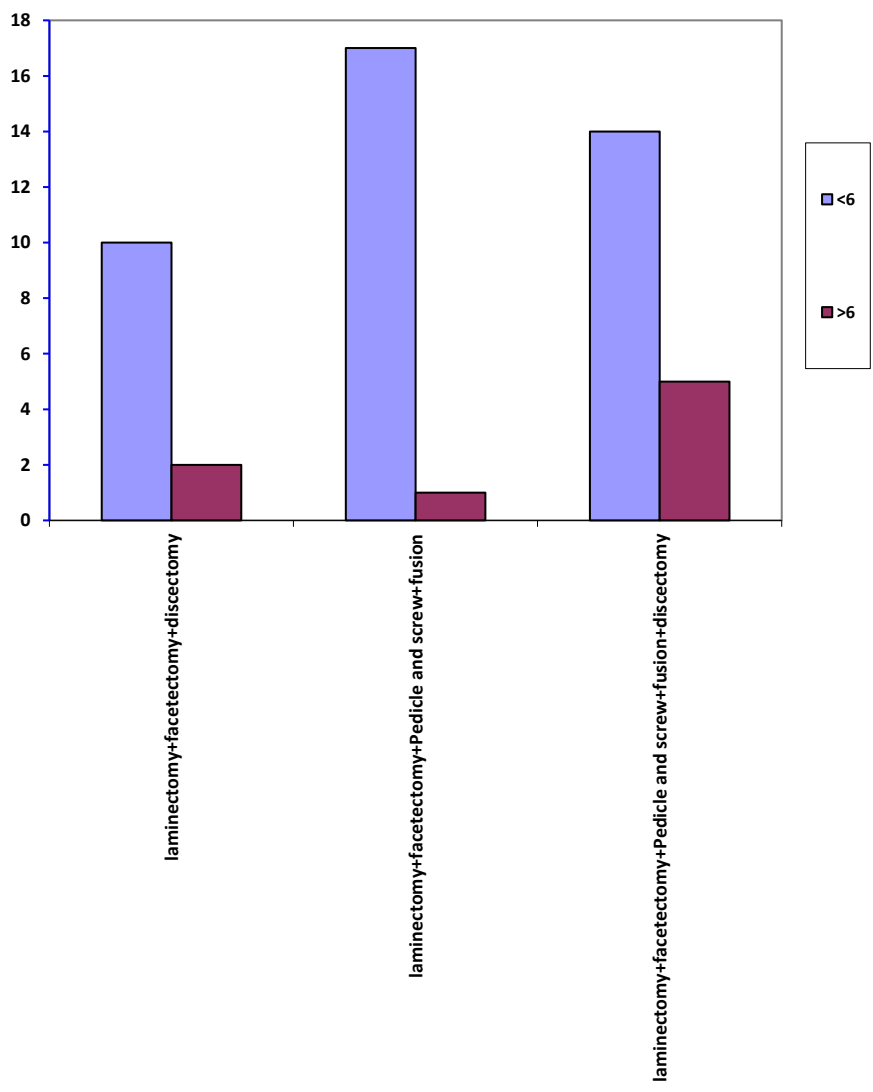

Figure 2. Comparison of frequency of lower limb pain in three different method of surgery based on VAS score 
dissectors, 24 (77.4\%) had a mild pain and 7 (22.6\%) had a severe limb pain. The relationship between dissectors and lower limb pain was not significant $(P=12.12)$. From 12 non-fusion patients, $10(83.3 \%)$ had a mild pain and $2(16.7 \%)$ had a severe lower extremity pain. From 37 patients with fusion, 31 (83.8\%) experienced a mild limb pain and $6(16.2 \%)$ a severe limb pain $(P=0.97)$. There was no significant relationship between fusion and lower extremity pain.

\section{Discussion}

Considering the low incidence of thoracolumbar disc disease (1\%), the results demonstrated that the prevalence of discs in this area was about $0.5 \%$ in the three studied medical centers. A small number (0.25\% to $5 \%$ ) of such patients are symptomatic; therefore, sufficient knowledge about the available options for surgical treatment is a matter of controversy. There are also no overall criteria for the optimum surgical procedure. Typical treatments involve dissectors through laminectomy. With regards to the potential complications of anterior and lateral approaches, all patients were surgically operated through the posterior surgery $[7,11]$.

In this study, out of 49 patients, 46 (93.9\%) experienced a decreased limb power before surgery, 29 (59.2\%) of whom showed a recovery of limb power after surgery. In 20 cases (40.8\%), the reduction of power persisted. The severity of postoperative limb weakness in our study was greater than those of Hyo Sang Lee $(1 \%)$ and Ning Liu (1\%) $[12,13]$. Moreover, limb weakness was greater than that of Jed S. Vanichkachorn, and Alexander R. Vaccaro (10.5\%) who had done with anterior surgery $[2,13]$. Due to the high degree of limb weakness before surgery $(93.9 \%)$ in our study and the late visit of the majority of patients more than one year after the onset of clinical symptoms, a greater limb weakness was observed.

In patients with discectomy, in the first and second groups, 31 (63.3\%) and 18 (36.7\%) patients experienced great lower limb pain, which was probably due to the cord traction for disc disassembly and manipulation and limited space for cord. Compared to the Ning Liu and Duk-Sung study, the results were almost identical. $[3,4,14]$. Therefore, minimum manipulation should be considered in the surgery of this area to the extent possible. In addition, in patients with dissectors (63.3\%), lower back pain was higher than those without dissectors (36.7\%).
In other words, a lesser degree of lower back pain was observed in the second group, which is probably due to neuron damages. Compared to the paresthesia of the limbs and perinea, before surgery (98\%) and postoperative surgery $(5.75 \%)$, it is true that we have the reduction in postoperative paresthesia, but compared with numerous articles $(10 \%)$ in our study were higher because of the fact that the time for referral to the doctor and the delay of patients in surgery even after the advice of physicians, and this has led to a much higher prevalence in our study (98\%) than in other studies $[3,4,15]$.

Back pain was $24.4 \%$ in the first group which not fused compare with $36.7 \%$ in the second and $24.4 \%$ in the third group. Overall, in this study, back pain was unlike the study of Ning Liu, in which the study eventually reduced back pain. Perhaps this was due to the use of inter body cage in addition to fusion and fixation with a pedicure screw, cause $80 \%$ of axial power is entered into the body of vertebral bone and in fusion without cage, it is possible to move and lack of fusion, whereas in this method we only fuse the posterior and medial column $[3,16,17]$.

Out of the 49 patients studied, 37 (75\%) underwent for fusion, no fusion was performed in 12 (25\%) patients, and the overall severity of back pain was higher in those who did not fusion (33.3\%) than in the fusion group (10.8\%) and more than the Ning Liu study (1\%), but in the Ning Liu study, inter body cage was used in addition to the screw and pedicle fixation and probably the cause of severe pain in the non-fusion group in our study due to instability and extra gestures in the operated area. Mild low back pain was in the non-fusion group $(66.7 \%)$, which was lower than that of the fusion group $(89.2 \%)$, which is probably due to greater field operation and greater soft tissue cut in fusion surgery, so we conclude that The less the extent of the area of operation, the less pain will have. Also, the amount of back pain in this study was much higher compared with the study by Jed S. Vanichkachorn and Alexander R.

Vaccaro, who had surgery with anterior approach, and had no reports of low back pain in their patients, so we conclude that the Para spinal muscles play a very important role in back pain and spinal dynamics of the spine, which, unfortunately, these muscles are damaged and fibroses in posterior approach $[2,3,18,19]$.

Out of the 49 patients studied, 37 (75\%) were fusion and $12(25 \%)$ were not fusion, and the severity of pain in the non-fusion group was $16.7 \%$ and in the fusion group 
$16.2 \%$, and the mild pain In the non-fusion group $83.3 \%$ and in the fusion group $83.8 \%$, it was interesting to note that there was no difference between the two groups regarding the amount of pain in the lower limb. Finally, the lower limb pain in our study was $16.3 \%$, which was more than Qi Q, Chen Qi (10.5\%) [5, 19-27].

\section{Conclusion}

Based on the results of this study, which examined the factors affecting prognosis and severity of pain after thoracolumbar discs, spinal cord manipulation should be avoided in this area due to limited circulation of the spinal area. In patients with hard discs, to discharge the disc and austerities, it is better to use posterior approach and fixation with a pedicure screw to reduce the severity of back pain in these patients and avoid excessive manipulation for dissectors.

There were no significant correlations between the variables, i.e. back pain and the three surgical methods, lower extremity pain and surgical technique, dissectors and lower limb pain, dissectors and back pain, fusion and fixation with back pain, fusion and fixation with lower limb pain. Due to the higher rates of complications in patients who had a history of myelopathy, they should be more cautious than normal patients. Overall, $61.2 \%$ of the patients were dissatisfied with their postsurgical life style, therefore, in the surgeries of this area, in choosing patients there should be more obsessive surgical complications and the likelihood of paresthesia remaining and worsening of back pain completely fully explained to patients.

\section{Ethical Considerations}

\section{Compliance with ethical guidelines}

All ethical principles were considered in this article. The participants were informed about the purpose of the research and its implementation stages; they were also assured about the confidentiality of their information; Moreover, They were allowed to leave the study whenever they wish, and if desired, the results of the research would be available to them.

\section{Funding}

This research did not receive any specific grant from funding agencies in the public, commercial, or not-forprofit sectors.

\section{Authors' contributions}

All authors contributed in designing, running, and writing all parts of the research.

\section{Conflict of interest}

The authors declared no conflict of interest.

\section{References}

[1] Lee HS, Shin DA, Yoon DH, Shin HC, Nyun K. Thoracolumbar junction disc herniation: Clinical features and surgical approaches. The Spine Journal. 2007; 4(3):129-33.

[2] Vanichkachorn, JS, Vaccaro AR. Thoracic disc disease: Diagnosis and treatment. Journal of the American Academy of Orthopaedic Surgeons. 2000; 8(3):159-69. [DOI:10.5435/00124635-200005000-00003] [PMID]

[3] Liu N. Chen Z. Qi Q, Li W, Guo Z. Circumspinal decompression and fusion through a posterior midline incision to treat central calcified thoracolumbar disc herniation: A minimal 2-year follow-up study with reconstruction CT. European Spine Journal. 2014; 23(2):373-81. [DOI:10.1007/s00586-0133054-4] [PMID] [PMCID]

[4] Kim DS, Lee JK, Jang JW, Ko BS, Lee JH, Kim SH. Clinical features and treatments of upper lumbar disc herniations. Journal of Korean Neurosurgical Society. 2010; 48(2):119-24. [DOI:10.3340/jkns.2010.48.2.119] [PMID] [PMCID]

[5] Qiang QI, Chen ZQ, Ning LI, Guo ZQ, Shi ZF, Liu ZJ, et al. Circumspinal decompression through a single posterior incision to treat thoracolumbar disc herniation. Chinese Medical Journal. 2011; 124(23):3852-7. [DOI:10.3760/cma.j.is sn.0366-6999.2011.23.004]

[6] Fogel GR, Toohey JS, Neidre A, Brantigan JW. Outcomes of L1-L2 posterior lumbar interbody fusion with the lumbar I/F cage and the variable screw placement system: reporting unexpected poor fusion results at L1-L2. The Spine Journal. 2006; 6(4):421-7. [DOI:10.1016/j.spinee.2005.09.011] [PMID]

[7] Eissa EM, Kersh AM. The application of transfacet pedicle sparing technique for removal of the herniated disc at the thoracic and upper lumbar Spine Egypt. Journal of Neurology, Neurosurgery, and Psychiatry. 2010; 47(4):605-10.

[8] Joaquim AF, Giacomini L, Ghizoni E, Fernandes FA, Mudo ML, Tedeschi H. Surgical anatomy and approaches to the anterior thoracolumbar spine region. Jornal Brasileiro de Neurocirurgia. 2013; 23(4):295-300

[9] Ritvo PG, Fischer JS, Miller DM, Andrews DW, LaRocca NG Multiple sclerosis quality of life inventory: A user's manual. New York: National Multiple Sclerosis Society; 2009.

[10] Hussain M, Raja RA, Makhdoom A. Ossification and hypertrophy of ligamentum flavum at thoracic spine. Journal of Ayub Medical College Abbottabad. 2014; 26(3):294-5. [PMID] 
[11] Amato V, Giannachi L, Irace C, Corona C. Thoracic spinal stenosis and myelopathy: Report of two rare cases and review of the literature. Journal of Neurosurgical Sciences. 2012; 56(4):373-8. [PMID]

[12] Oppenlander ME, Clark JC, Kalyvas J, Dickman CA. Surgical management and clinical outcomes of multiple-level symptomatic herniated thoracic discs. Journal of Neurosurgery: Spine. 2013; 19(6):774-83. [DOI:10.3171/2013.8.SPI NE121041] [PMID]

[13] Li M, Wang Z, Du J, Luo Z, Wang Z. Thoracic myelopathy caused by ossification of the ligamentum flavum: A retrospective study in Chinese patients. Clinical Spine Surgery. 2013; 26(1):E35-40. [DOI:10.1097/BSD.0b013e31827ada68] [PMID]

[14] Cornips EM, JanssEn ML, Beuls EA. Thoracic disc herniation and acute myelopathy: Clinical presentation, neuroimaging findings, surgical considerations, and outcome. Journal of Neurosurgery:Spine. 2011; 14(4):520-8. [DOI:10.3171/2010.12. SPINE10273] [PMID]

[15] Yamasaki R, Okuda S, Maeno T, Haku T, Iwasaki M, Oda T. Surgical outcomes of posterior thoracic interbody fusion for thoracic disc herniations. European Spine Journal. 2013; 22(11):2496-503. [DOI:10.1007/s00586-013-2877-3] [PMID] [PMCID]

[16] Arnold PM, Johnson PL, Anderson KK. Surgical management of multiple thoracic disc herniations via a transfacet approach: A report of 15 cases. Journal of Neurosurgery: Spine. 2011; 15(1):76-81. [DOI:10.3171/2011.3.SPINE10642] [PMID]

[17] Mori K, Imai S, Kasahara T, Nishizawa K, Mimura T, Matsusue $Y$. Prevalence, distribution, and morphology of thoracic ossification of the posterior longitudinal ligament in Japanese: Results of CT-based cross-sectional study. Spine. 2014; 39(5):394-9. [DOI:10.1097/BRS.0000000000000153] [PMID]

[18] Koda M, Furuya T, Okawa A, Inada T, Kamiya K, Ota M, et al. Mid-to long-term outcomes of posterior decompression with instrumented fusion for thoracic ossification of the posterior longitudinal ligament. Journal of Clinical Neuroscience. 2016; 27:87-90. [DOI:10.1016/j.jocn.2015.07.027] [PMID]

[19] Yin H, Shi H. Concurrent ossification of posterior longitudinal ligament and ossification of ligamentum flavum in the thoracic spine demonstrated by SPECT/CT imaging. Clinical Nuclear Medicine. 2015; 40(3):228-30. [DOI:10.1097/ RLU.0000000000000689] [PMID]

[20] Feng FB, Sun CG, Chen ZQ. Progress on clinical characteristics and identification of location of thoracic ossification of the ligamentum flavum. Orthopaedic Surgery. 2015; 7(2):8796. [DOI:10.1111/os.12165] [PMID]

[21] Yamazaki M, Okawa A, Fujiyoshi T, Furuya T, Koda M Posterior decompression with instrumented fusion for thoracic myelopathy caused by ossification of the posterior longitudinal ligament. European Spine Journal. 2010; 19(5):691-8. [DOI:10.1007/s00586-009-1266-4] [PMID] [PMCID]

[22] Wang YQ, Liu XG, Jiang L, Jiang L, Feng WE, Miao YU, et al. Intraoperative ultrasonography in "cave-in" 360 circumferential decompression for thoracic spinal stenosis. Chinese Medical Journal. 2011; 124(23):3879-85. [DOI:10.3760/cma.j.is sn.0366-6999.2011.23.009]

[23] Machino M, Yukawa $Y$, Ito K, Nakashima H, Kato F A new thoracic reconstruction technique "transforaminal thoracic interbody fusion": A preliminary report of clini- cal outcomes. Spine. 2010; 35(19):E1000-5. [DOI:10.1097/ BRS.0b013e3181dc9153] [PMID]

[24] Ito Z, Matsuyama Y, Ando M, Kawabata S, Kanchiku T, Kida K, et al. Postoperative paralysis from thoracic ossification of posterior longitudinal ligament surgery risk factor of neurologic injury: Nationwide multiinstitution survey. Spine. 2016; 41(19):E1159-63. [DOI:10.1097/BRS.0000000000001585] [PMID]

[25] Arts MP, Bartels RH. Anterior or posterior approach of thoracic disc herniation? A comparative cohort of mini-transthoracic versus transpedicular discectomies. The Spine Journal. 2014; 14(8):1654-62. [DOI:10.1016/j.spinee.2013.09.053]

[26] Nacar OA, Ulu MO, Pekmezci M, Deviren V. Surgical treatment of thoracic disc disease via minimally invasive lateral transthoracic trans/retropleural approach: Analysis of 33 patients. Neurosurgical Review. 2013; 36(3):455-65. [DOI:10.1007/s10143-013-0461-2]

[27] Matsumoto M, Toyama Y, Chikuda H, Takeshita K, Kato $\mathrm{T}$, Shindo $\mathrm{S}$, et al. Outcomes of fusion surgery for ossification of the posterior longitudinal ligament of the thoracic spine: A multicenter retrospective survey. Journal of Neurosurgery: Spine. 2011; 15(4):380-5. [DOI:10.3171/2011.6.SPINE10816] [PMID] 
This Page Intentionally Left Blank 